\title{
Approach to optimization of FRAP methodology for studies based on selected monoterpenes
}

\author{
Karolina A. Wojtunik-Kulesza
}

\begin{abstract}
Terpenes, wide-spread secondary plant metabolites, constitute an important part of natural compounds with various biological activity. Among them are substances revealing antioxidant, calming, antiviral, or analgesic activities. Due to terpenes' high volatility and their low solubility in water, studies on these compounds prove to be difficult and relevant methodology needs to be adjusted to specific character of compounds being examined. Considering significant influence of iron ions on dementia development, terpenes' activity towards reduction of $\mathrm{Fe}^{3+}$ should be determined. To date, methodology of FRAP has not been adapted to terpenes, thus, results obtained were unreliable. Taking this fact into account, the aim of the studies was optimization of the method for monoterpenes. Studies included three modifications: 1. slight adjustment of entire FRAP procedure; 2. replacing methanol with other solvents (heptane, butanone, ethyl acetate); 3. addition of Tween 20. Obtained results revealed a significant improvement of reduction activity of selected terpenes (linalool, $\alpha$-phellandrene, $\alpha$-terpinene) in studies with Tween 20, whereas replacing methanol with other solvents did not produce expected effects.
\end{abstract}

Keywords: terpenes; FRAP; iron ions; optimization; secondary plant metabolites 